\title{
Perceptual difficulty differences predict asymmetry in redundant modification with color and material adjectives
}

\author{
Leyla Kursat \& Judith Degen*
}

\begin{abstract}
When referring to objects, speakers are often more specific than necessary for the purpose of establishing unique reference, e.g., by producing redundant modifiers. A computational model of referring expression production that accounts for many of the key patterns in redundant adjectival modification assumes that adjectives differ in how noisy (reliable), and consequently, how useful they are for reference. Here we investigate one hypothesis about the source of the assumed adjectival noise: that it reflects the perceptual difficulty of establishing whether the property denoted by the adjective holds of the contextually relevant objects. In Exp.1, we collect perceptual difficulty norms for items that vary in color and material. In Exp. 2, we test the highest (material) and lowest (color) perceptual difficulty items in a reference game and find that material is indeed less likely to be mentioned redundantly, replicating previous work. In Exp. 3, we obtain norms for the tested items in a second perceptual difficulty measure with the aim of testing the effect of perceptual difficulty within property type. The overall results provide preliminary support for the hypothesis that the propensity to redundantly use color over material adjectives may be driven by the relative ease of assessing an object's color, compared to the relative difficulty of assessing its material.
\end{abstract}

Keywords. reference; perception; overinformativeness; redundancy; experimental pragmatics

1. Introduction. Speakers often include redundant modifiers in referring expressions (Pechmann 1989; Gatt et al. 2011; Arts et al. 2011; Koolen et al. 2013). This redundancy is typically termed overmodification because it appears to violate the Gricean Quantity-2 maxim to say no more than necessary. However, the systematic structure in adjectival redundancy has recently been argued to be the result of a linguistic system geared towards efficient communication (Degen et al. 2020; Rubio-Fernandez et al. 2019). One way in which variability in the production of redundant referring expressions is structured is through asymmetries in the redundant use of color, size and material adjectives. When size or material is sufficient for singling out the intended referent, speakers routinely include redundant color adjectives in their utterances (e.g., "the green plastic chair" instead of "the plastic chair" in Fig. 1). However, in contexts where color is sufficient for unique reference, speakers rarely mention size or material redundantly (Pechmann 1989; Sedivy 2003; Gatt et al. 2011; Rubio-Fernandez 2016; Degen et al. 2020). Moreover, speakers' knowledge of the typicality of the properties of objects and the features of the context interact with these asymmetries. Color adjectives are more likely to be produced redundantly with increasing scene variation (Degen et al. 2020; Davies \& Katsos 2013; Koolen et al. 2013) and adjectives are more likely the be produced redundantly, the more atypical the property denoted by the adjective is for the object under discussion (Degen et al. 2020; Westerbeek et al. 2015; Mitchell et al. 2013).

\footnotetext{
* We are grateful to Chaz Firestone for helpful discussion of the notion of perceptual difficulty, and for inspiring the design of Exp. 3. Authors: Leyla Kursat, Stanford University (lkursat@stanford.edu) \& Judith Degen, Stanford University (jdegen@stanford.edu)
} 


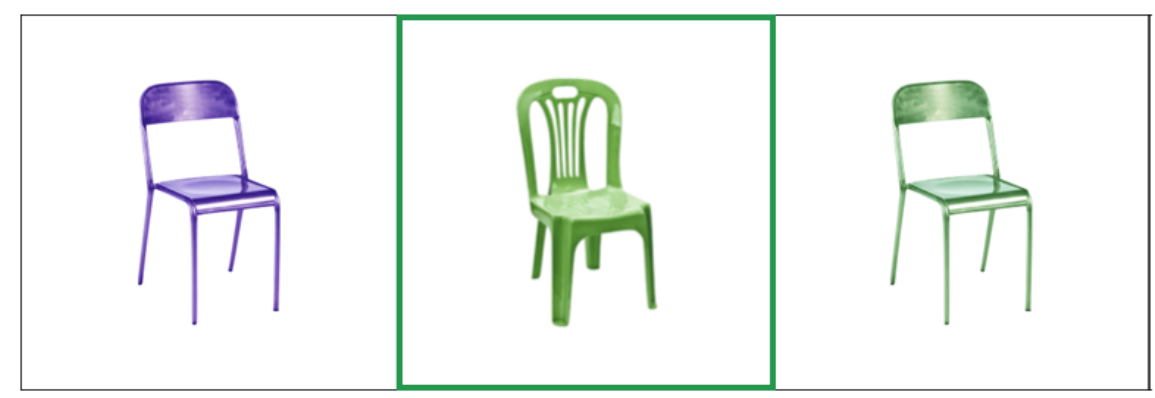

Figure 1. Example context where material is sufficient for unique reference. Intended referent is marked by the green border.

There are many accounts of redundancy in referring expressions, including pragmatic, semantic, lexical and visual ones. The pragmatic account proposed by Sedivy (2003) builds on the contrastive effect of mentioning the color of objects that occur in predictable colors. According to this account, highly predictable properties of objects are not encoded as part of these objects' default descriptions and therefore, their use triggers a contrastive inference and provides referential disambiguation. Although this account qualitatively captures typicality effects that have been shown to modulate redundant modification, it does not explain the source of these defaults in referential communication, nor when speakers depart from them. In addition to listeners' expectations of informativity, production of overinformative referring expressions has been shown to depend on the object category (Rubio-Fernandez 2016) and the semantics of the adjective involved (Rubio-Fernandez et al. 2019; Sedivy 2003). Relatedly, based on a comparison of eye movement patterns in the online comprehension of color and scalar adjectives, Aparicio et al. (2018) report that processing differences may be the attributable to differences in the semantics of the adjectives, i.e., the result of differences in processing relative vs. absolute adjectives. However, explanations of adjectival production asymmetries that rest on differences in the semantics of the adjectives do not capture the fine-grained contextdependence of redundant modification. For instance, Viethen et al. (2017) showed that a decrease in color contrast between displayed objects reduces the use of color adjectives in referring expressions. Accounts that focus on the role of perceptual factors in redundant modification take into consideration such contextual effects and argue that redundant adjective use is sensitive to the relative visual salience of properties adjectives denote (Tarenskeen et al. 2015; Rubio-Fernandez et al. 2019; Jara-Ettinger \& Rubio-Fernandez 2020), such that speakers choose utterances with considerations of their perceptual utility for the listener. That is, speakers are not only aiming to be informative but rather efficient in their utterance choices, in order to facilitate the listener's target search.

In this paper, we explore a hypothesis aligned with accounts of the production of referring expression that center the communicative efficiency of perceptually salient properties of objects (Jara-Ettinger \& Rubio-Fernandez 2020), motivated by a recent computational model of referring expression production that spells out why redundant referring expressions may be more communicatively efficient than their non-redundant counterparts (Degen et al. 2020). Couched within the Rational Speech Act (RSA) framework (Goodman \& Frank 2016), this model treats speakers and listeners as agents recursively reasoning about each other's mental states to communicate. The simple RSA model assumes that objects have deterministic lexical meanings 
and that speakers choose utterances that maximize informativeness with respect to those meanings. This model doesn't generate overinformative referring expressions mainly because it calculates the informativeness (and cost) of mentioning the redundant property to be equal to the informativeness of mentioning the alternative (only mentioning the sufficient property). Degen et al. (2020) extend this model by focusing on the calculation of informativeness and relaxing the Boolean semantics to a non-deterministic continuous semantics that returns real values between 0 and 1. By allowing utterances to be informative about objects to varying degrees, this continuous semantics assumes that adjectives differ in how noisy or unreliable, and consequently, how useful they are for the purpose of establishing reference. The noisier the adjective, the less useful it is for allowing a listener to infer the intended referent. In contrast, the more reliable the adjective, the more useful it is even when used redundantly, because it allows for restricting the domain of reference more faithfully than the noisier adjectives do. Degen et al. (2020) show that assuming size adjectives are noisier than color adjectives accounts for asymmetries in the redundant production of color vs. size adjectives, and effects of scene complexity on redundant modification fall out of the model for free. However, this account raises the crucial question regarding the source of the presumed adjectival noise. The authors speculatively raise a few possibilities for what the noise might reflect. For instance, it could represent the perceptual difficulty of establishing whether an object exhibits the property denoted by the adjective. Alternatively, it might reflect the past communicative success in using a particular adjective type. It may also reflect the agent's prior beliefs about the correlations between features of objects.

Here, we take the first step in testing the first possibility, which we spell out in the Perceptual Difficulty Hypothesis: that the noise term reflects the perceptual difficulty of establishing whether the property denoted by the adjective holds of the contextually relevant objects in the domain of color and material adjectives. The prediction is that systematic differences in the redundant modification patterns observed for color and material adjectives can be explained by a difference in perceptual difficulty of establishing whether objects are of a particular color or material. The more difficult it is to judge whether an object has a property, the less likely speakers should be to redundantly mention that property. In Exp.1, we norm the perceptual difficulty associated with establishing whether an object exhibits a color or material and select the highest and lowest perceptual difficulty objects for testing in Exp. 2. In Exp. 2, we test in an interactive reference game whether adjectives that denote more perceptually difficult property - material - are indeed less frequently produced redundantly than the perceptually easier to establish color. Finally, in Exp. 3, we replicate the perceptual difficulty measures from Exp. 1 and investigate the role of perceptual difficulty within property type.

2. Experiment 1: Measuring perceptual difficulty. First, we collected perceptual difficulty norms for assessing the color and material of 81 images. Through a timed forced choice task we measured the out-of-context perceptual difficulty of establishing whether objects have a particular color or are made of a particular material.

2.1. PARTiCipAnts. We recruited 120 participants through Amazon Mechanical Turk. We excluded participants who were self-reported non-native English speakers $(n=4)$ and participants with accuracy lower than $75 \%(n=11)$.

2.2. PROCEDURE. Participants saw images of objects alongside a color or material adjective and were asked to indicate whether the object had the property denoted by the adjective or not. 
Their task was to indicate "yes" or "no" by pressing the F or J key as quickly as possible. If participants did not respond within 4 seconds, the trial timed out. When participants responded correctly, a green border appeared around their selection, and when they responded incorrectly a red border appeared.

We collected perceptual difficulty norms for 12 objects that each occurred in two or three different materials and in three different colors. All resulting 81 images were separately normed for object nameability, property nameability, object typicality and property typicality. Every participant saw each image once and we collected 30 judgements for each image with matching and non-matching color and material adjectives. Color and material adjectives that didn't match the images were randomly selected for each participant from a pool of adjectives denoting properties of other images in the experiment.

2.3. RESUlTS. Figure 2 shows the proportion and response times of correct responses to color and material adjectives. In order to assess whether material is more perceptually difficult to assess than color, we conducted two types of analyses. First, we analyzed the responses by conducting a mixed effects logistic regression predicting the log odds of making an error from a dummy-coded fixed effect of property type (reference level: 'color'). Second, we analyzed the response times of correct responses by conducting a mixed effects linear regression predicting log-transformed response time from property type. Both models included the maximal random effects structure justified by the design: by-item and by-participant intercepts and by-participant slopes for property type. Overall, material adjectives resulted in higher error rates $(\beta=0.48, S E=0.12, p<.0001)$ and greater response times $(\beta=5.44, S E=4.74, \mathrm{t}=11.49$, $p<.0001$ ) than color adjectives, suggesting that material is indeed a more perceptually difficult property to establish than color. These results provide preliminary evidence for the Perceptual Difficulty Hypothesis.

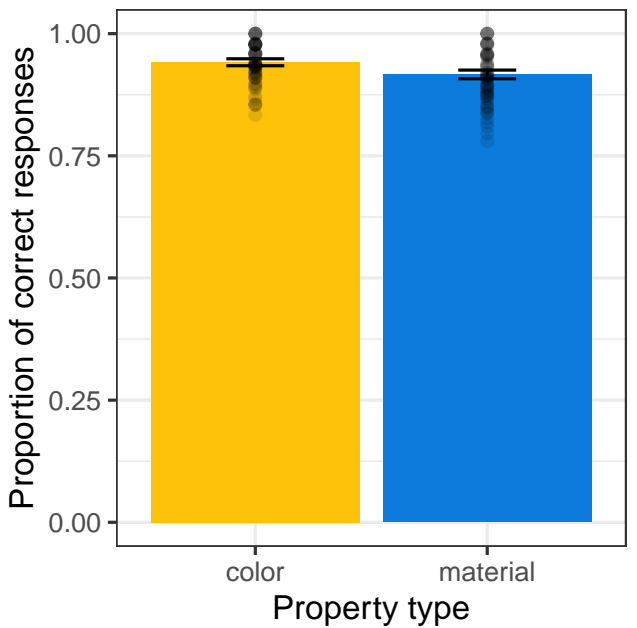

(a) Proportion of correct responses.

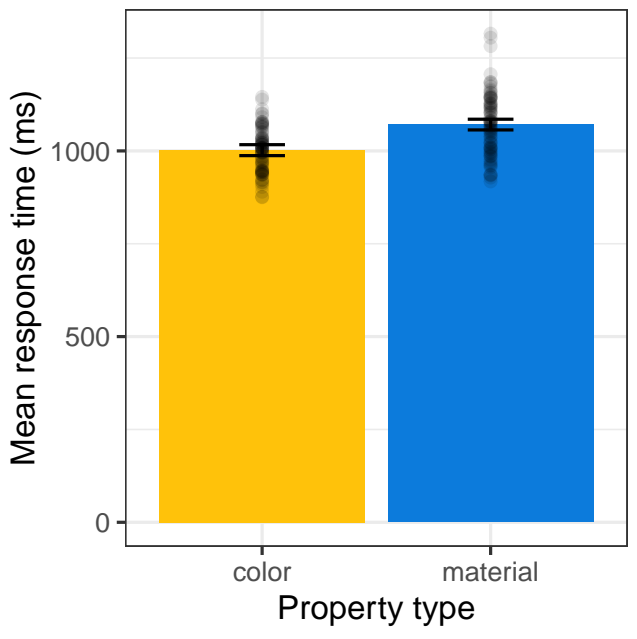

(b) Response times for correct responses.

Figure 2. Responses to color and material adjectives in Experiment 1. Individual dots represent participant means and error bars indicate $95 \%$ bootstrapped confidence intervals.

In order to determine a set of items to use for the production study in Exp. 2, we selected two sets of items from the total set of normed items: a high difficulty set of perceptually diffi- 
cult image-adjective pairs and a low difficulty set of perceptually less difficult image-adjective pairs. To create these sets, we first ordered the image-word combinations along six different measures: (1) response time regardless of response correctness (correct, incorrect) and regardless of response type (yes, no), (2) response time for only correct responses, regardless of response type, (3) response time for only correct 'yes' responses, (4) response time for only correct 'no' responses, (5) error rate regardless of response type, (6) error rate only among 'yes' responses (7) error rate only among 'no' responses. Image-material adjective pairs were consistently among the highest difficulty items and image-color adjective pairs were consistently among the lowest difficulty items. We thus selected the 8 image-material adjective pairs with the consistently highest error rate and response times for the high difficulty set, and the 8 image-color adjective pairs with the consistently lowest error rate and response times for the low difficulty set. We used these final 16 items as target items in Exp. 2.

3. Experiment 2: Production of referring expressions. The goal of Exp. 2 was to measure the production probability of redundant color and material adjectives for the items normed in Exp. 1. In a free production interactive reference game we tested whether the less perceptually difficult color was more frequently produced redundantly than the more perceptually difficult material. ${ }^{1}$

3.1. Participants. We recruited 100 participants through Amazon Mechanical Turk and randomly paired them into speaker-listener dyads to play a real time communication game (50 pairs, Hawkins 2015). We excluded games where participants reported a native language different from English $(\mathrm{n}=5)$.

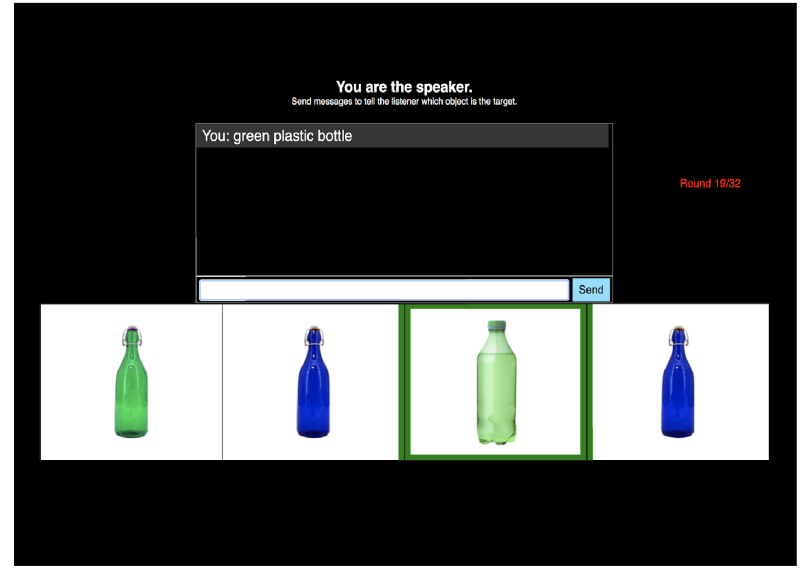

Figure 3. Example display from Exp. 2: speaker's perspective on a low-difficulty (color redundant) trial.

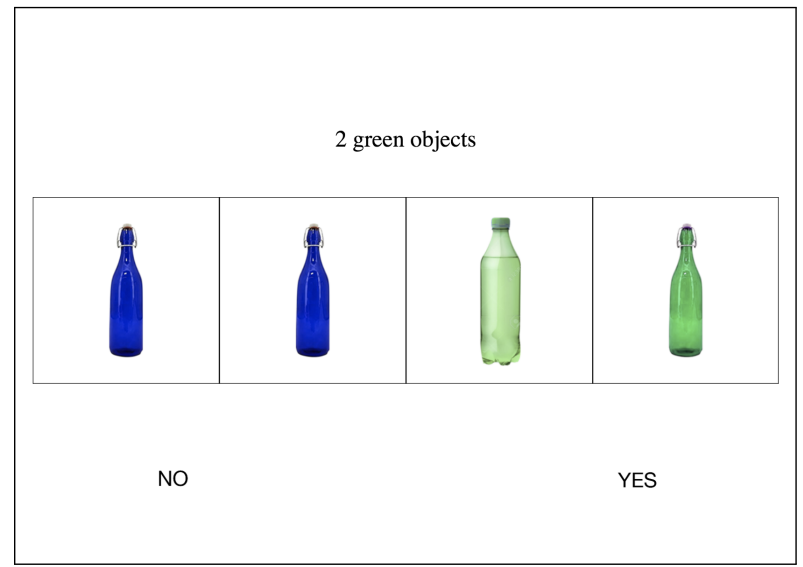

Figure 4. Example display from Exp. 3: color trial with correct number.

3.2. Procedure. On each trial, participants saw a display with 4 images and chat box. Both the speaker and the listener saw the same images in different positions. One of the images was designated as the target image, and marked by a green border in the speaker's display. The

${ }^{1}$ This experiment was preregistered at https://osf.io/57c6u. Experimental materials, data, and analysis files for all three experiments can be accessed at https://github.com/leylakursat/perceptual_difficulty. 
speaker's task was to describe this target image to the listener using the chat box to send messages. The listener's task was to guess the target image by clicking. After the listener made a selection, both participants received feedback about whether the target image was selected and advanced to the next trial.

Participants completed 32 trials. Of these, half were critical trials and half were filler trials. On critical trials (Figure 3), the 4 images were of the same object type (e.g., 4 bottles) and either color or material was redundant for distinguishing the target. One of the images, the competitor, always shared the redundant property with the target and the two distractors shared the sufficient property with the competitor. ${ }^{2}$ On 8 high-difficulty / material redundant trials, mentioning the material was redundant; on 8 low-difficulty / color redundant trials, color was redundant. On filler trials, the 4 images contained different objects and both color and material were redundant. Filler items were of 4 different types: the competitor either shared the color, material, both or none of the properties with the target.
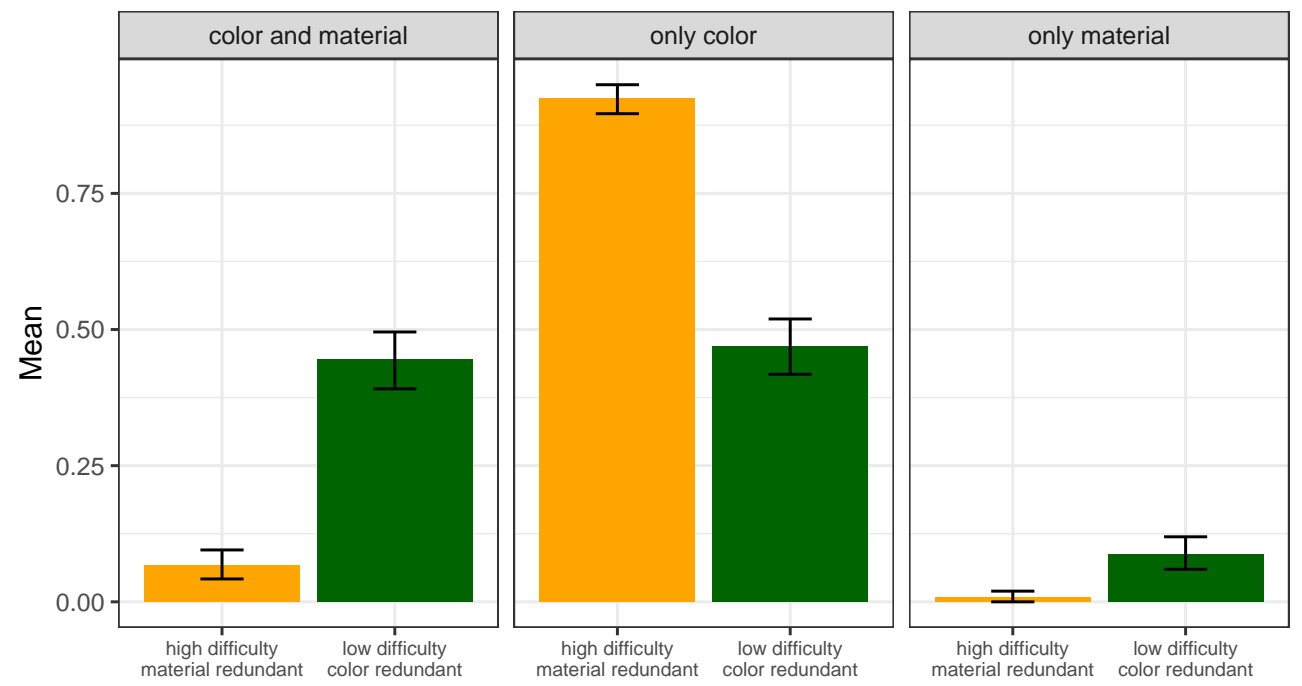

Figure 5. Proportion of redundant "color and material" utterances vs. non-redundant utterances on high and low difficulty trials. Error bars indicate $95 \%$ bootstrapped confidence intervals

3.3. RESUltS AND DISCUSSION. To investigate the use of redundant adjectives, we first classified the produced utterances as redundant "color and material" (e.g., the blue metal chair), minimal or underinformative "only color" (e.g., the green cup) and minimal or underinformative "only material" (e.g., the metal chair). Proportion of redundant and non-redundant referring expressions are shown in Figure 5.

To assess whether color was more likely to be mentioned redundantly than material, we conducted a mixed effects logistic regression predicting redundant adjective use from a dummycoded fixed effect of redundant property (reference level: 'color'), with random by-subject and by-item intercepts and slopes for redundant property. There was a main effect of redundant property, such that speakers were more likely to redundantly mention color than material

2 This was one of the conditions identified by Degen et al. (2020) as resulting in a high probability of redundant modification. 
( $\beta=2.32, S E=0.64, p<.0001)$, replicating the previously observed asymmetry between redundant modification with color and material adjectives on a new set of items (Sedivy 2005; JaraEttinger \& Rubio-Fernandez 2020). Our analysis of the responses to filler trials showed that the preference to mention color transferred to trials in which neither color nor material mention was required for unique reference.
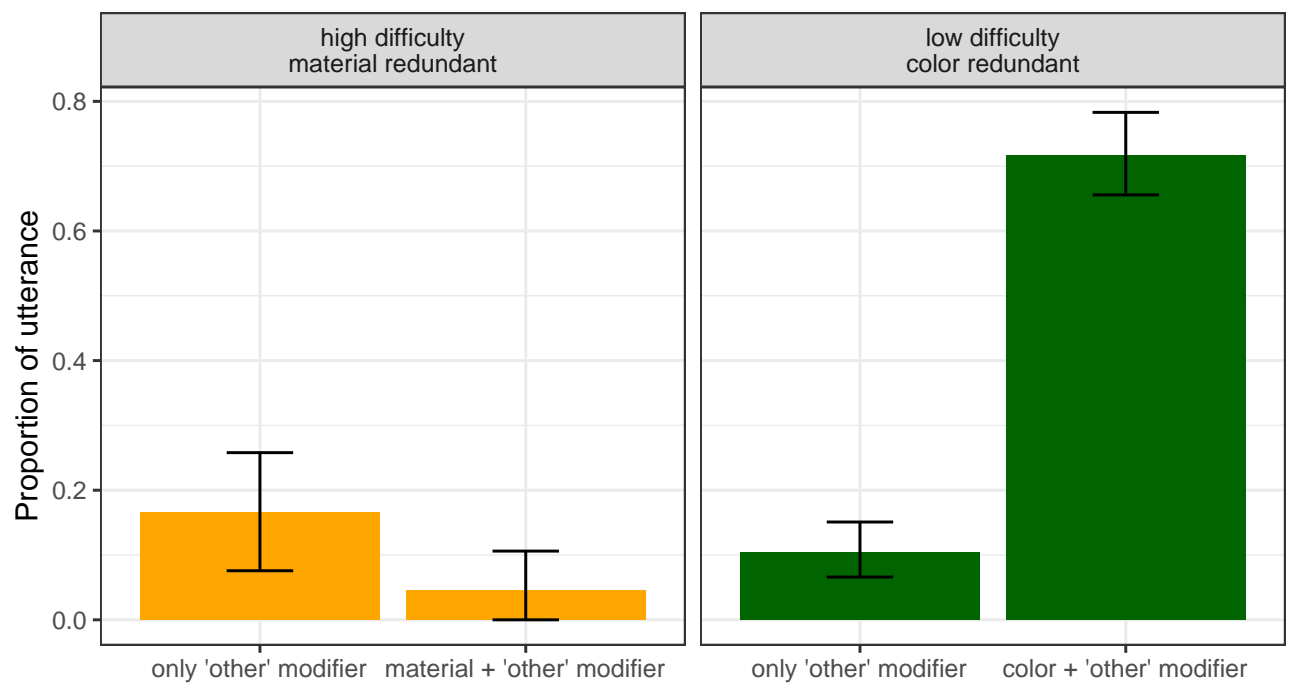

Figure 6. Proportion of utterance of "other" modifier types with modifier denoting the redundant property. Error bars indicate $95 \%$ bootstrapped confidence intervals

What is not visible in Figure 5 is that on $39 \%$ of trials, participants mentioned modifiers other than the expected color and material adjectives to refer to the target. These modifiers included shape (rectangular table, square table), size (long table, short boot), shade (dark blue plate, light green vase) and other (solo cup, shiny pitcher) modifiers. To assess whether the use of other modifiers contains any additional evidence for an asymmetry in preference for using color versus material adjectives, Figure 6 shows the distribution of "other" modifiers produced instead of the sufficient adjective, i.e., the proportion of trials on which speakers preferred to avoid mentioning the sufficient property altogether. While the rate of other modifier uses was generally low, an exception is the color redundant condition, where material would have been sufficient for identifying the referent: in this case, participants frequently avoided producing the material modifier by instead mentioning color together with another property that correlated with the material, e.g., shiny blue table instead of metal table, or by modifying color directly, e.g., dark blue plate. These results provide further evidence that participants dispreferred mentioning material.

Together with the norms from Exp. 1, these results suggest that the more difficult it is to judge whether an object has a property, the less likely speakers are to redundantly mention that property, providing further support for the Perceptual Difficulty Hypothesis. However, a stronger test of the hypothesis would be to ask whether within-property differences in perceptual difficulty are predictive of within-adjective-type variability in redundant modification; e.g., whether an easier-to-perceive material is mentioned redundantly more frequently than a harderto-perceive material. Unfortunately, the within-property differences in perceptual difficulty for 
the tested items were very small on both the error measure and the response time measure elicited in Exp. 1. Moreover, the items in this study were specifically selected for maximal perceptual difficulty differences between color and material. The combination of the very low amount of variability in perceptual difficulty within property and the relatively stark separation of color and material in perceptual difficulty space thus does not afford us the power to test the stronger version of the Perceptual Difficulty Hypothesis with the data we have reported thus far. Exp. 3 aims to remedy part of this problem by obtaining perceptual difficulty estimates for the same items with a more sensitive perceptual difficulty measure that is likely to yield greater within-property variability.

4. Experiment 3: Perceptual difficulty in context. The goal of Exp. 3 was to obtain an additional measure of perceptual difficulty for the items used in Exp. 2, both to replicate the results of Exp. 1 on a second measure and to subject the Perceptual Difficulty Hypothesis to a stronger test by probing within-property perceptual difficulty effects on redundant adjective use measured in Exp. 2.

4.1. PARTICIPANTS. We recruited 400 participants through Prolific. We excluded participants with accuracy lower than $75 \%(n=24)$ and responses that were 2.5 standard deviations away from the mean response time ( $2 \%$ of all trials).

4.2. Procedure. Exp. 3 was identical to Exp. 1 but instead of seeing the images in isolation, participants saw the displays from the production experiment (Exp. 2). These displays appeared with short descriptions that were of the form "X [adjective] objects" and included a number and either a color or material adjective (see Figure 4). Participants were asked to press a button as quickly as possible to indicate whether the display contained the described objects or not. For example, in Fig. 4, "2 green objects" was true but "2 plastic objects" was false. On half of the trials, the statement was correct and on the other half, it was incorrect. The use of color and material adjectives was also balanced. We collected 100 judgements for each property in the display, for all the different displays. Each participant completed 31 trials.

4.3. RESULTS. Figure 7 shows the proportion and response times of correct responses to color and material adjectives. We first report analyses to assess whether the results of Exp. 1 replicate on this perceptual difficulty task. We then report analyses aimed at testing the strong version of the Perceptual Difficulty Hypothesis.

As in Exp. 1, in order to assess whether material is more perceptually difficult to assess than color, we conducted two types of analyses. First, we analyzed the responses by conducting a mixed effects logistic regression predicting the log odds of making an error from a dummycoded fixed effect of property type (reference level: 'color'). Second, we analyzed the response times of correct responses by conducting a mixed effects linear regression predicting log-tranformed response time from property type (reference level: 'color'). Both models included the maximal random effects structure justified by the design: by-item and by-participant intercepts and by-participant slopes for property type. Overall, material adjectives resulted in higher error rates $(\beta=0.96, S E=0.09, p<.0001)$ and greater response times $(\beta=0.24, S E=0.018$, $\mathrm{t}=-59.62, p<.0001)$ than color adjectives, replicating the results of Exp. 1.

To address the strong version of the Perceptual Difficulty Hypothesis, namely that perceptual difficulty modulates redundancy above and byeond property type, an additional analysis step was necessary. Because property type is a strong predictor of response time and property 


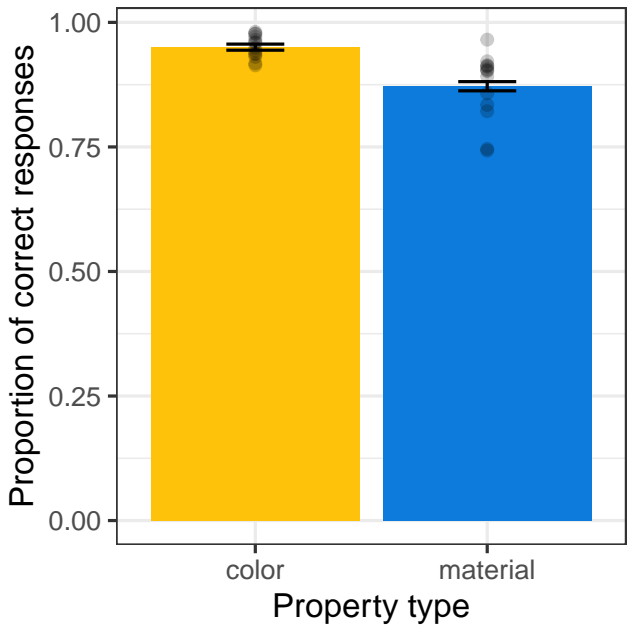

(a) Proportion of correct responses.

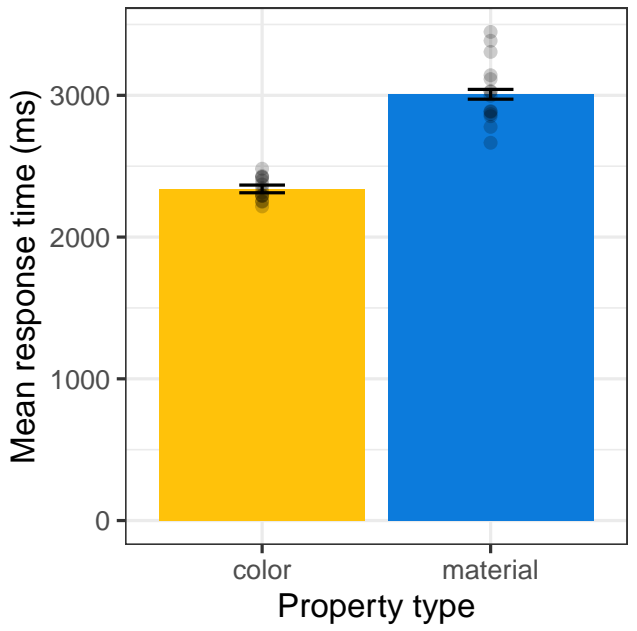

(b) Response times for correct responses.

Figure 7. Responses to color and material adjectives in Experiment 3. Individual dots represent participant means and error bars indicate $95 \%$ bootstrapped confidence intervals

type and response time would be highly collinear in a regression analysis, we first regressed log-transformed response times against property type using a simple linear model. We then used the residuals of this model as the predictor in a mixed effects logistic regression predicting redundant adjective use in Exp. 2 from the mean-centered predictors property type, perceptual difficulty (i.e., residualized response time), and their interaction. We replicated the effect of property type on response time $(\beta=7.43, S E=2.27, p<.001)$ but found neither a main effect of perceptual difficulty $(\beta=-11.3, S E=16.41, p=.49)$ nor an interaction between property type and perceptual difficulty $(\beta=13.57, S E=31.87, p=.67)$ on redundant adjective use. This analysis suggests that there is no evidence for within-property effects of perceptual difficulty on redundant adjective use.

To further test the strong version of the Perceptual Difficulty Hypothesis, we used another measure of perceptual difficulty: the relative perceptual difficulty of assessing the sufficient property compared to the redundant property. We computed the log ratio of mean sufficient property response time to mean redundant property response time and performed the same residualization step described above. Again, there was no significant effect of perceptual difficulty above and beyond property type $(\beta=5.31, S E=3.87, p=.17))$.

5. General discussion. We tested the role of perceptual difficulty in explaining the asymmetry in redundant color and material adjective use in referring expressions. The prediction generated by the Perceptual Difficulty Hypothesis is that the more difficult it to establish whether an object is of a particular color or material, the less likely speakers should be to redundantly mention that property.

There are two versions of the Perceptual Difficulty Hypothesis. The work thus far provides evidence for the weak version of this hypothesis, that the propensity to redundantly use color and material adjectives may be driven by the asymmetry in the perceptual difficulty involved in establishing whether or not a particular object is of a particular color or material. Exp. 1 and Exp. 3 both provide support for this hypothesis, since in both experiments color 
adjectives generally resulted in lower error rates and lower response times than material adjectives. Under the strong version of the hypothesis, perceptual difficulty should modulate redundancy within property type, such that, e.g., easier-to-perceive material should be mentioned redundantly more frequently than harder-to-perceive material. The data collected thus far does not provide evidence for the strong version of the hypothesis. This may be either because the strong version does not hold, or for a simpler, methodological reason: the items we selected were structured such that between-property differences in perceptual difficulty were maximized, and within-property differences were minimized. Follow-up work should assess the strong version of the hypothesis on a separate set of items that includes perceptual difficulty overlap between color and material as well as greater within-property variability in perceptual difficulty.

It is possible that the effects of perceptual difficulty on redundant adjectival modification are confounded by the retrieval difficulty of the adjectives, in line with availability-based production theories (Bock 1987; Ferreira \& Dell 2000). That is, it may be that material adjectives are mentioned less frequently simply because they are harder to retrieve from memory, in turn perhaps because they are less frequent in the input. A cursory corpus search makes this possibility unlikely: in the Google Books Ngram Viewer (based on a corpus of over 20 million print books), the material noun combinations used in our experiments were more frequent than the color noun combinations, showing that retrieval formalized in terms of frequency, can't account for these results.

The results overall are consistent with efficiency-based accounts of redundant adjective use (Rubio-Fernandez 2016; Degen et al. 2020) and provide preliminary evidence that the noise term in Degen et al. (2020)'s computational model of referring expression production might be a function of the perceptual difficulty involved in property verification. However, these results do not rule out other possibilities for what the noise term might reflect, including past communicative utility of adjectives. One avenue of future work is to use explicit computational models to test to what extent the average success of using certain adjective types is a predictor of their redundant use.

The formalization of contextual informativeness in Degen et al. (2020)'s model is neutral in regards to whether redundant modification is a speaker-internal or listener-oriented process (Arnold 2008). While some researchers argue that redundant modification helps the speaker because the redundant attributes are more easily produced (Davies \& Katsos 2013; Koolen et al. 2013), others argue that it helps the listener by making it easier to identify the target (Fussell \& Krauss 1989; Arts et al. 2011; Rubio-Fernandez 2016; Rehrig et al. 2021). Our results cannot adjudicate between these two views. We provided evidence that speakers prefer to redundantly mention less perceptually difficult properties, but this may be either because it aids the addressee in target identification or because it aids the speaker in retrieval.

The results also raise an interesting issue for the study of redundant modification crosslinguistically: if perceptual difficulty involved in property verification is indeed the driving force behind redundant modification, similar patterns of redundant modification should be observed in languages besides English, assuming that perceptual mechanisms are shared across people. However, recent work by Rubio-Fernandez et al. (2020) and Wu \& Gibson (2021) show that word order-in particular, whether a language has pre- or post-nominal adjectivesis a predictor of cross-linguistic differences in redundant modifier use. Differences in redundant modification are attributed to the way in which incremental production planning inter- 
acts with language-specific word order facts. Recent modeling work derives asymmetries in redundant adjectival modification cross-linguistically by combining Degen et al. (2020)'s noisy semantics with Cohn-Gordon et al. (2018)'s incrementality mechanism within RSA (Waldon $\&$ Degen 2021). However, the cross-linguistic empirical picture is far from clear and requires much future work. A particularly interesting domain in this regard is afforded by the study of redundant modification in emerging languages: if perceptual difficulty involved in property verification is indeed the driving force behind redundant modification, then in the absence of established referring conventions, redundant modification should be even more strongly driven by the perceptual factors discussed here.

In sum, we have provided evidence that perceptual factors are a likely contributing factor in explaining speakers' propensity to produce redundant referring expressions. While this result alone is compatible with a purely speaker-internal production pressure mechanism, the mounting empirical evidence instead points towards a communicative system geared towards optimizing communicative efficiency, within which considerations of perceptual difficulty are just one of many factors contributing to production choices.

\section{References}

Aparicio, Helena, Christopher Kennedy \& Ming Xiang. 2018. Perceived informativity and referential effects of contrast in adjectivally modified NPs. In Elena Castroviejo, Louise McNally \& Galit Weidman Sassoon (eds.), The semantics of gradability, vagueness, and scale structure, 199-220. Dordrecht: Springer.

Arnold, Jennifer E. 2008. Reference production: Production-internal and addressee-oriented processes. Language and Cognitive Processes 23(4). 495-527.

https://doi.org/10.1080/01690960801920099.

Arts, Anja, Alfons Maes, Leo Noordman \& Carel Jansen. 2011. Overspecifica- tion facilitates object identification. Journal of Pragmatics 43(1). 361-374. https://doi.org/10.1016/j.pragma.2010.07.013.

Bock, Kathryn. 1987. Exploring levels of processing in sentence production. In Gerard Kempen (ed.), Natural language generation, 351-363. Dordrecht: Springer. https://doi.org/10.1007/978-94-009-3645-4_22.

Cohn-Gordon, Reuben, Noah D. Goodman \& Christopher Potts. 2018. An incremental iterated response model of pragmatics. Proceedings of the Society for Computation in Linguistics 2(1).

Davies, Catherine \& Napoleon Katsos. 2013. Are speakers and listeners 'only moderately Gricean'? An empirical response to Engelhardt et al. (2006). Journal of Pragmatics 49(1). 78-106. https://doi.org/10.1016/j.pragma.2013.01.004.

Degen, Judith, Robert D. Hawkins, Caroline Graf, Elisa Kreiss \& Noah D. Goodman. 2020. When redundancy is useful: A Bayesian approach to "overinformative" referring expressions. Psychological Review 127(4). 591-621. https://doi.org/10.1037/rev0000186.

Ferreira, Victor S. \& Gary S. Dell. 2000. Effect of ambiguity and lexical availability on syntactic and lexical production. Cognitive Psychology 40(4). 296-340. https://doi.org/10.1006/cogp.1999.0730.

Fussell, Susan R. \& Robert M. Krauss. 1989. The effects of intended audience on message production and comprehension: Reference in a common ground framework. Journal of Experimental Social Psychology 25(3). 203-219. https://doi.org/10.1016/0022-1031(89)90019-X. 
Gatt, Albert, Roger P. G. van Gompel, Emiel Krahmer \& Kees van Deemter. 2011. Nondeterministic attribute selection in reference production. In Kees van Deemter, Albert Gatt, Roger van Gompel \& Emiel Krahmer (eds.), Proceedings of the Workshop on the Production of Referring Expressions (PRE-CogSci 2011), 1-7. Boston, MA: Cognitive Science Society.

Goodman, Noah D. \& Michael C. Frank. 2016. Pragmatic language interpretation as probabilistic inference. Trends in Cognitive Sciences 20(11). 818-829. https://doi.org/10.1016/j.tics.2016.08.005.

Hawkins, Robert X. D. 2015. Conducting real-time multiplayer experiments on the web. Behavior Research Methods 47(4). 966-976. https://doi.org/10.3758/s13428-014-0515-6.

Jara-Ettinger, Julian \& Paula Rubio-Fernandez. 2020. The social basis of referential communication: Speakers construct reference based on listeners' expected visual search. https://doi.org/10.31234/osf.io/fzuvh.

Koolen, Ruud, Martijn Goudbeek \& Emiel Krahmer. 2013. The effect of scene variation on the redundant use of color in definite reference. Cognitive Science 37(2). 395-411. https://doi.org/10.1111/cogs.12019.

Mitchell, Margaret, Ehud Reiter \& Kees Van Deemter. 2013. Typicality and object reference. Proceedings of the Annual Meeting of the Cognitive Science Society 35. 3062.

Pechmann, Thomas. 1989. Incremental speech production and referential overspecification. Linguistics 27(1). 89-110.

Rehrig, Gwendolyn, Reese A Cullimore, John M. Henderson \& Fernanda Ferreira. 2021. When more is more: Redundant modifiers can facilitate visual search. Cognitive Research: Principles and Implications 6(1). 1-20.

Rubio-Fernandez, Paula. 2016. How redundant are redundant color adjectives? An efficiencybased analysis of color overspecification. Frontiers in Psychology 7. 153. https://doi.org/10.3389/fpsyg.2016.00153.

Rubio-Fernandez, Paula, Francis Mollica \& Julian Jara-Ettinger. 2020. Speakers and listeners exploit word order for communicative efficiency: A cross-linguistic investigation. Journal of Experimental Psychology: General. 150(3). 583-594. https://doi.org/10.1037/xge0000963.

Rubio-Fernandez, Paula, Helena Aparicio Terrasa, Vishakha Shukla \& Julian Jara-Ettinger. 2019. Contrastive inferences are sensitive to informativity expectations, adjective semantics and visual salience. PsyArXiv. Preprint. https://doi.org/10.31234/osf.io/mr4ah.

Sedivy, Julie C. 2003. Pragmatic versus form-based accounts of referential contrast: Evidence for effects of informativity expectations. Journal of Psycholinguistic Research 32(1). 3-23. https://doi.org/10.1023/A:1021928914454.

Sedivy, Julie C. 2005. Evaluating explanations for referential context effects: Evidence for Gricean meachanisms in online language interpretation. In John C. Trueswell \& Michael K. Tanenhaus (eds.), Approaches to studying world-situated language use: Bridging the language-as-product and language-as-action traditions, 345-364. Cambridge, MA: MIT Press.

Tarenskeen, Sammie, Mirjam Broersma \& Bart Geurts. 2015. Overspecification of color, pattern, and size: salience, absoluteness, and consistency. Frontiers in Psychology 6. 1703. https://doi.org/10.3389/fpsyg.2015.01703.

Viethen, Jette, Thomas van Vessem, Martijn Goudbeek \& Emiel Krahmer. 2017. Color in reference production: the role of color similarity and color codability. Cognitive Science 41. 1493-1514. https://doi.org/10.1111/.ogs.12387. 
Waldon, Brandon \& Judith Degen. 2021. Modeling cross-linguistic production of referring expressions. Proceedings of the Society for Computation in Linguistics 4(1). 206-215. https://doi.org/10.7275/vsfn-t057.

Westerbeek, Hans, Ruud Koolen \& Alfons Maes. 2015. Stored object knowledge and the production of referring expressions: The case of color typicality. Frontiers in Psychology 6. 935. https://doi.org/10.3389/fpsyg.2015.00935.

Wu, Sarah A. \& Edward Gibson. 2021. Word order predicts cross-linguistic differences in the production of redundant color and number modifiers. Cognitive Science 45(1). e12934. https://doi.org/10.1111/cogs.12934. 